\title{
Advantages and limitations of clinical guidelines: Can we change clinicians' behaviour?
}

\author{
ALFONSO MELE $^{1}$ and GIOVANNI DE GIROLAMO ${ }^{2}$ \\ ${ }^{\prime}$ Center of Epidemiology, Health Surveillance and Promotion, Italian National Institute of Health, Rome (Italy) \\ ${ }^{2}$ Health Care Research Agency, Emilia-Romagna Region, Bologna (Italy)
}

\section{VARIATIONS IN HEALTH CARE PATTERNS}

Findings from health service studies conducted in Italy, and from other in-depth investigations of health care patterns conducted in this country and in Europe (e.g., pharmacoepidemiological research on the quantitative and qualitative characteristics of drug prescription patterns) all point to a marked lack of homogeneity in health care delivery indicators. Indeed, this result is likely the most salient lesson gleaned from several studies comparing different services and facilities over an entire nation, such as the national survey of residential facilities (PROGRES) (de Girolamo et al., 2002) and the national survey of acute, public and private inpatient facilities (PROGRES-Acute) (de Girolamo et al., 2007). Interestingly, this variability also represents the main finding of the guidelines implementation SIEP-DIRECT'S Project (DIscrepancy between Routine practice and Evidence in psychiatric Community Treatments on Schizophrenia) recently promoted and conducted by the Italian Society of Epidemiological Psychiatry's, presented in this Monographic issue of EPS (Ruggeri, 2008; Semisa et al., 2008; Ruggeri et al., 2008).

The observed differences in quantitative and qualitative service indicators (e.g., facility number and location, size, treatment characteristics, etc.) are due to marked inter-regional diversity in mental health service planning and delivery - the consequence of the high

Address for correspondence: Dr. G. de Girolamo, Agenzia Sanitaria e Sociale Regionale, Viale Aldo Moro 21, 40121 Bologna (Italy). Fax: +39-051-6397053

E-mail: gdegirolamo@regione.emilia-romagna.it Declaration of Interest: None. degree of autonomy afforded in Italy to Regions by the recent federal (re)organization of the nation. Most of these variations have been found to have no explanations in terms of varying incidence or prevalence rates of mental disorders. For instance, in the PROGRES survey the ratio of residential beds between the two regions of Abruzzo and Campania was 10:1; in the following study (PROGRES-Acute), the ratio of inpatient, public and private acute psychiatric beds between Calabria and Umbria was 8.5:1. Clearly, variations of this amplitude are unrelated to the prevalence of severe mental disorders in these regions, and are due only to divergent health policies of various, regional and local health authorities. Needless to say, such high variability in indicator type can lead to inequality and disparity in terms of access and use of services for citizens living in different Regions (but in the same country). These variations can be little or not at all influenced or modified by individual clinicians' input, as practitioners generally remain passive spectators of political decisions that are generally made with little or no consultation of available data.

Another major source of variation in the area of health care is that of clinicians' diagnostic and treatment-related decisions. There is now overwhelming evidence showing that different clinicians frequently make quite different, even opposite and unreconcilable decisions when assessing the same patient or the same clinical scenario - phenomenon that has also has precise cognitive explanations (Klein, 2005).

EBM proponents have suggested that a clinician's individual performance is the result of the interaction between expertise (not be confused with experience!) and motivation, divided by the amount (and strength) of institutional barriers. Hence, in addition to institutional constraints, which can have a powerful influence on

Epidemiologia e Psichiatria Sociale, 17, 4, 2008 
clinicians' behaviour, this diagnostic and treatment decision variability also concerns differences in expertise, motivation, training background, time available for clinical practice, etc; and cognitive patterns of decisionmaking represent the final pathway of this complex array of interactive variables. It can be easily ascertained that the key variable of expertise varies among practitioners. As highlighted by Geddes et al. (1998), "There are only 2 explanations for these variations: either there is no evidence on which to base practice, or that there is evidence, but at least some of us are not using it. The inevitable result of these variations is that some patients are not receiving the best available care”.

In this perspective guidelines can do nothing to increase or restore individual motivation, and can only help remove certain institutional barriers: their main objective is to improve individual clinicians' expertise and to help them be better equipped in terms of scientific knowledge, and perhaps more psychologically confident of the soundness of their own decisions. One can assume that the achievement of this objective will lead to a more homogeneous and evidence-based style of health care delivery.

The purpose of guidelines should therefore be that of helping practitioners base their diagnostic and treatmentrelated decisions on the results of methodologically wellgrounded experimental and observational research and reducing the area of 'subjective', opinion-based decisions.

\section{HOW TO CHANGE CLINICIANS' BEHAVIOUR?}

Given that the ultimate aim of a guideline is to trigger and foster change in clinicians' diagnostic and treatment decisions in the effort to improve patient outcomes, we must first ascertain whether this objective can actually be achieved, and if so, in what ways.

Yet, changing and improving clinicians' performance and laying the groundwork for the optimal utilization of available evidence are aims that are sought not only through clinical guideline implementation. In fact, a wide range of educational strategies have also been developed and tested (see Table I, adapted from Grol \& Grimshaw; 2003). The cited authors have recently published a comprehensive analysis of barriers and facilitators to change practice and have assessed the effectiveness of different dissemination and implementation strategies; in their contribution, they used and then updated 54 previously published reviews. Given that guidelines can be disseminated or promoted only through the strategies they examined, it is crucial to evaluate their effectiveness: Table 1 shows the main conclusions drawn by the two authors for each of the 17 strategies or intervention programmes. The overall conclusion is that there is no simple way to translate guideline-summarised evidence into clinical practice. Some methods seem to be more effective than others, and change is possible when a well-designed intervention programme is used. Interestingly, most of the programmes examined did show a positive influence on clinicians' behaviour and skills, but the effect size was rather small. At the same time, however, none of the intervention programmes examined seemed more effective of other strategies for change in all settings. The results of Grol \& Grimshaw's review (2003) are in line with findings from other systematic reviews (Perkins et al., 2007; Thomson O'Brien et al., 2001), which have consistently shown that single-focus interventions are rarely effective in promoting visible and durable change, and that only multifaceted interventions are most effective - i.e., programmes combining small group educational activities, financial incentives, sanctions, interventions by recognized opinion leaders, individual detailing (that is, use of specific officers, like drug-industry information officers, but paid by the NHS), feedback and audit, reminders, and introduction of computers into daily clinical practice. Notably, the two most common forms of intervention (e.g., dissemination of printed material and course or conference attendance) have been found to be the least effective in promoting change. It is also important to note that in Italy, multifaceted interventions, such as the abovedescribed approach, have only rarely ever been attempted and (to the best of our knowledge) never in the field of mental health care.

We conclude this section with Grol and Grimshaw's final words in their Lancet review: "If you would like to start tomorrow to change practice and implement evidence, prepare well: involve the relevant people; develop a proposal for change that is evidence based, feasible, and attractive; study the main difficulties in achieving the change, and select a set of strategies and measures at different levels linked to that problem; of course, within your budget and possibilities. Define indicators for measurement of success and monitor progress continuously or at regular intervals. And, finally, enjoy working on making patients' care more effective, efficient, safe, and friendly". Their proposal is exciting, but certainly not an easy task! 
Table I - Overview of strategies for implementation of evidence and improvement in clinicians' performances (adapted from Grol \& Grimshaw, 2003).

\begin{tabular}{ll}
\hline TYPE OF STRATEGY OR INTERVENTION & GENERAL OUTCOMES \\
\hline Educational materials & Mixed effects \\
Conferences, courses & Mixed effects \\
Interactive small group meetings & Mostly effective, but limited numbers of studies \\
Educational outreach visits & Especially effective for prescribing/prevention \\
Use of opinion leaders & Mixed effects \\
Education with different educational strategies & Mixed effect s, dependent on combination of strategies \\
Feedback on performance & Mixed effects, most effective for test ordering \\
Reminders & Mostly effective, particularly for prevention \\
Computerised decision support & Mostly effective for drug dosing and prevention \\
Introduction of computers in practice & Mostly effective \\
Substitution of tasks & Pharmacist: effect on prescribing nurse: mixed effects \\
Multiprofessional collaboration & Effective for a range of different chronic conditions \\
Mass media campaigns & Mostly effective \\
Total quality management/continuous quality improvement & Limited effects, mostly single-site non-controlled studies \\
Financial interventions & Fundholding and budgets effective, mainly on prescribing \\
Patient-mediated interventions & Mixed effects; reminding by patients is effective in prevention \\
Combined interventions & Most reviews: more effective than single interventions; \\
& not confirmed in recent reviews.
\end{tabular}

\section{GUIDELINES IN THE ITALIAN HEALTH CARE SYSTEM}

Having briefly described the general context of guideline dissemination and implementation, mention should be made of the actions and steps taken over the last few years by the Italian National Health Service to foster the production, dissemination, and implementation of clinical guidelines in particularly relevant public health areas previously lacking 'good clinical practice' indicators.

The Italian government's response to the issue has recently led to several concrete actions, one of which was the establishment of the Sistema di Verifica e Controllo sull'Assistenza Sanitaria (SiVeAS), a national audit and control plan for the Italian health care system. This programme in turn has generated many other initiatives, including an agreement between the Ministry of Health and the National Institute of Health (Istituto Superiore di Sanità - ISS): the Sistema Nazionale Linee Guida $(S N L G)$, i.e., National Health Care System Guidelines. The SNLG has the overall aim of promoting the production and implementation of guidelines as an effective National Health Care System clinical governance instrument; the agreement therefore calls for the development of a number of clinical practice guidelines.

Guideline topics are commissioned by the Ministry of Health on the basis of national health priorities, and each guidelines is updated every 3-4 years, unless the need to do so arises earlier. Two consensus conferences are also held for each guideline topic.
The main steps involved in clinical guideline development are as follows:

- Establishment of a multidisciplinary panel whose aim is to discuss key questions, define inclusion/exclusion criteria, and identify the specific terms required to construct the literature search strategy;

- A systematic review of the literature using an explicit search strategy for each clinical question;

- Formulation of evidence- based recommendations.

Modifying clinical behaviour on the basis of the best evidence available entails a complex implementation process. Context analysis to identify barriers to change represents the first step in designing effective implementation strategies. A targeted implementation strategy including single or multiple interventions such as educational intervention programmes, audit and feedback loops can then be activated.

To date, the SNLG has funnelled most of its implementation resources into the widespread diffusion of produced guidelines, and on the above-mentioned information activities. Constructing evaluation activities represents the next step to be accomplished. Intervention may be evaluated ex ante and ex post using appropriate indicators, and to this end, we note that a list of indicators with related data sources is provided towards the end of each SNLG guideline. Comprehensive and effective implementation activities, however, cannot yet be undertaken, due to the SNLG programme's limited budget and to the organizational structure of the Italian NHS. 
It is therefore our hope that strategies to further promote the dissemination, implementation, and evaluation of evidence-based guidelines will be enhanced in the future, so to improve the quality of mental health care and hence the satisfaction of patients and their family members. The SIEP-DIRECT'S Project presented in this Issue is an important step in this process.

\section{REFERENCES}

de Girolamo G., Picardi A., Micciolo R., Falloon I., Fioritti A. \& Morosini P. for the Italian PROGRES study group (2002). Residential care in Italy: a national survey of non-hospital facilities. British Journal of Psychiatry 181, 220-225.

de Girolamo G., Barbato A., Bracco R., Gaddini A., Miglio R., Morosini P., Norcio B., Picardi A., Rossi E., Rucci P., Santone G., \& Dell'Acqua G. (2007). The characteristics and activities of acute psychiatric inpatient facilities: A national survey in Italy. British Journal of Psychiatry 191, 170-177.

Geddes J., Reynolds S., Streiner D., Szatmari P. \& Haynes B. (1998). Evidence-based practice in mental health. Evidence Based in Mental Health 1, 4-5.

Grol R. \& Grimshaw J. (2003). From best evidence to best practice: effective implementation of change in patients' care. Lancet 362 , 1225-1230.
Klein J.G. (2005). Five pitfalls in decisions about diagnosis and prescribing. British Medical Journal 330, 781-783.

Perkins M.B., Jensen P.S., Jaccard J., Gollwitzer P., Oettingen G., Pappadopulos E. \& Hoagwood K.E. (2007). Applying theory-driven approaches to understanding and modifying clinicians' behavior: what do we know? Psychiatric Services 58, 342-348.

Ruggeri M. (2008). Guidelines for treating mental illness: love them, hate them. Can the SIEP-DIRECT'S Project serve in the search for a happy medium? Epidemiologia e Psichiatria Sociale 17(4), 270-277.

Ruggeri M., Lora A. \& Semisa D. on behalf of the SIEP-DIRECT'S Group (2008). The SIEP-DIRECT'S Project on the discrepancy between routine practice and evidence. An outline of the main findings and the practical implications for the future of community based mental health services. Epidemiologia e Psichiatria Sociale 17(4), 358-368.

Semisa D., Lora A., Morosini P. \& Ruggeri M. (2008). The SIEPDIRECT'S Project on the discrepancy between routine practice and evidence in the treatment of schizophrenia. The design, the indicators, and the methodology of the study (in Italian). Epidemiologia e Psichiatria Sociale 17(4), 278-290.

Thornicroft G. \& Tansella M. (2007). The Mental Health Matrix: A Manual to Improve Services. Cambridge University Press: Cambridge.

Thomson O'Brien M.A., Freemantle N., Oxman A.D., Wolf F., Davis D.A. \& Herrin J. (2001). Continuing education meetings and workshops: effects on professional practice and health care outcomes. Cochrane Database Systematic Reviews Issue 2, CD003030. 